Pesq. Vet. Bras. 35(1):49-54, janeiro 2015

DOI: $10.1590 / \mathrm{S} 0100-736 \mathrm{X} 2015000100011$

\title{
Neoplasmas oculares e de anexos em cães e gatos no Rio Grande do Sul: 265 casos (2009 -2014) ${ }^{1}$
}

\author{
Kivia L. Hesse ${ }^{2}$, Gabriela Fredo ${ }^{2}$, Lorena L.B. Guimarães ${ }^{2}$, Matheus O. Reis ${ }^{2}$, João \\ A.T. Pigatto ${ }^{3}$, Saulo P. Pavarini ${ }^{2}$, David Driemeier ${ }^{2}$ e Luciana Sonne ${ }^{2 *}$
}

\begin{abstract}
Hesse K.L., Fredo G., Guimarães L.L.B., Reis M.O., Pigatto J.A.T., Pavarini S.P., Driemeier D. \& Sonne L. 2015. [Ocular and annexes neoplasms in dogs and cats in Rio Grande do Sul: cases 265 (2009-2014).] Neoplasmas oculares e de anexos em cães e gatos no Rio Grande do Sul: 265 casos (2009-2014). Pesquisa Veterinária Brasileira 35(1):4954. Setor de Patologia Veterinária, Faculdade de Veterinária, Universidade Federal do Rio Grande do Sul, Av. Bento Gonçalves 9090, Prédio 42505, Porto Alegre, RS 91540-000, Brazil. E-mail: luciana.sonne@ufrgs.br

Ocular neoplasms cause discomfort, loss of vision or may also reflect systemic diseases. A retrospective study has been performed to identify and analyze ocular and annexes neoplasms obtained by biopsies sent to the Sector of Veterinary Pathology at the Federal University of Rio Grande do Sul (SPV/ UFRGS). 265 diagnoses of ocular and annexes neoplasms, $87.5 \%$ in canine species and $12.5 \%$ in feline species, were made from January 2009 to December 2014. Neoplasms occurred more in animals over the age of 12 months and animals of old age, dogs (52/232) and cats (21/33) mixed breed were the most affected. The eyelid was most commonly affected in dogs $(164 / 232)$ and feline $(20 / 33)$, followed by the third eyelid in dogs $(20 / 232)$ and eye orbit in cats (5/33). The most common tumor diagnosed was meibomian adenoma in dogs $(82 / 232)$ and squamous cell carcinoma in cats $(10 / 33)$ both in the eyelids. Twenty four tumor types have been identified in dogs and 16 in cats. Benign neoplasms in dogs represented the largest number of diagnosis (56\%) whereas in cats, there were more cases of malignant neoplasms $(75.8 \%)$.
\end{abstract}

INDEX TERMS: Dog, cat, neoplasm, eye, eyelid.

RESUMO.- Neoplasias oculares causam desconforto, problemas de visão e podem refletir doenças sistêmicas. Um estudo retrospectivo foi realizado para identificar e analisar neoplasmas oculares e de anexos obtidos por biópsias enviadas ao Setor de Patologia Veterinária (SPV) da Universidade Federal do Rio Grande do Sul (UFRGS). Durante o período de janeiro de 2009 a dezembro de 2014 realizou-se 265 diagnósticos de neoplasmas oculares e de anexos, destes $87,5 \%$ na espécie canina e em $12,5 \%$ na espécie felina. As neoplasias ocorreram mais em animais com idade superior a 12 meses e idosos, e os cães (52/232) e gatos $(21 / 33)$ sem raça definida foram os mais acometidos.

\footnotetext{
${ }^{1}$ Recebido em 15 de janeiro de 2015.

Aceito para publicação em 30 de janeiro de 2015.

${ }^{2}$ Setor de Patologia Veterinária, Faculdade de Veterinária, Universidade Federal do Rio Grande do Sul (UFRGS), Av. Bento Gonçalves 9090, Prédio 42505, Porto Alegre, RS 91540-000, Brasil. *Autor para correspondência: luciana.sonne@ufrgs.com.br

${ }^{3}$ Hospital de Clínicas Veterinárias, Faculdade de Veterinária, UFRGS, Av. Bento Gonçalves 9090, Porto Alegre, RS 91540-000.
}

A pálpebra foi o local mais acometido na espécie canina (164/232) e felina (20/33), seguida pela terceira pálpebra em cães (20/232) e órbita nos felinos (5/33). 0 tumor mais diagnosticado nos cães foi o adenoma meibomiano $(82 / 232)$ e nos gatos o carcinoma de células escamosas (10/33), ambos em pálpebras. Foram identificados nos cães 24 tipos tumorais e nos gatos 16 . Em caninos as neoplasias benignas representaram o maior número de diagnósticos (56\%) sendo que em felinos o maior número de casos foi de neoplasias malignas $(75,8 \%)$.

TERMOS DE INDEXAÇ̃̃O: Cão, gato, neoplasma, olho, pálpebra.

\section{INTRODUÇÃO}

Neoplasias oculares são importantes enfermidades da oftalmologia veterinária. Essas quando presentes no bulbo ocular, órbita ou anexos podem ter consequências para a visão, aparência e conforto do animal, bem como ser um sinalizador de doença grave sistêmica. Em virtude de sua localização, até mesmo neoplasias oculares benignas po- 
dem causar cegueira e perda do olho (Miller \& Dubielzig 2007).

Os neoplasmas oculares podem ser classificados em primários e secundários sendo que os primários são frequentes em cães e gatos (Wilcock 2007b), e normalmente exibem um baixo potencial metastático (Conceição et al. 2010). Esses tumores são uma causa comum de enucleação em gatos (Dubielzig 2011) e a segunda maior causa de enucleação em cães (Wilcock, 2007a).

Ao exame macroscópico estas neoplasias se caracterizam por massas e nódulos, com tamanhos e colorações variadas, conforme sua classificação histológica. Além disso, inflamação e glaucoma secundário podem surgir a partir de neoplasias e causar um dano severo ao olho (Conceição et al. 2010). Esse trabalho tem por objetivo identificar e analisar a ocorrência das neoplasias oculares e dos seus anexos em cães e gatos através do estudo retrospectivo de amostras enviadas ao Setor de Patologia Veterinária (SPV) da Universidade Federal do Rio Grande do Sul (UFRGS) no período de janeiro de 2009 a dezembro de 2014.

\section{MATERIAL E MÉTODOS}

Para o estudo foram analisados os arquivos de diagnóstico de biópsias oculares enviadas ao Setor de Patologia Veterinária (SPV) da Universidade Federal do Rio Grande do Sul (UFRGS) no período de janeiro de 2009 a dezembro de 2014. Foram avaliados casos de cães e gatos diagnosticados com neoplasia ocular e de anexos. As amostras eram procedentes do Hospital de Clínicas Veterinárias da UFRGS e clínicas veterinárias da Região Metropolitana de Porto Alegre, Rio Grande do Sul, Brasil. Os registros de diagnósticos anatomopatológicos foram analisados para identificar dados gerais dos animais, tipo neoplásico, localização anatômica e identificação de neoplasmas malignos e benignos.

\section{RESULTADOS}

No período de janeiro de 2009 a dezembro de 2014 foram processadas e diagnosticadas 25.800 biópsias de cães e gatos, dessas, 314 correspondem a amostras oculares de cães e gatos, totalizando $1,21 \%$ das biópsias recebidas no SPV-UFRGS. Desse total, 265 casos (84,4\% das biópsias) corresponderam a lesões neoplásicas, $27(8,6 \%)$ a lesões de origem infecciosa e $22(7,0 \%)$ a alterações congênitas, traumáticas e hiperplásicas. Dos 265 casos de lesões neoplásicas, 232 (87,5\%) eram de cães e 33 (12,5\%) de gatos.

A faixa etária observada na espécie canina foi de 5 meses a 18 anos com média de idade de 8,4 anos. A espécie felina apresentou faixa etária que variou de 3 a 20 anos com média de 11 anos. Os cães (48/232) SRD foram os mais afetados, seguidos pelas raças Poodle (21/232), Labrador (19/232) e Dachshund (14/232) e houve alguns casos onde a raça não foi informada $(9 / 232)$. Os gatos SRD $(22 / 33)$ foram os mais acometidos e as únicas raças catalogadas foram a Persa $(3 / 33)$ e a Siamesa $(2 / 33)$ e casos onde a raça não foi informada (6/33). Nos Quadros 1 e 2 estão discriminados os diagnósticos de neoplasias oculares e de anexos em cães e gatos, respectivamente, sendo estes divididos conforme a sua localização e ocorrência. Nos cães e gatos foram registrados 24 e 16 neoplasmas diferentes, respectivamente. Em caninos as neoplasias benignas re- presentaram o maior número de diagnósticos (56\%) sendo que em felinos o maior número de casos foi de neoplasias malignas $(75,8 \%)$.

O número de fêmeas caninas $(121 / 232)$ foi semelhante ao número de machos $(111 / 232)$, porém em felinos $66,67 \%$ dos casos de neoplasias foram diagnosticados em fêmeas (22/33) e machos representaram $33,33 \%$ dos diagnósticos $(11 / 33)$.

Os seis principais tumores diagnosticados nos caninos foram o adenoma meibomiano (82/232), o melanoma $(35 / 232)$, o hemangiossarcoma (15/232), o epitelioma meibomiano (14/232), o CCE (12/232) e o melanocitoma $(12 / 232)$. As localizações mais frequentemente acometidas nos cães foram a pálpebra (164/232), a 3 3 pálpebra (20/232) e a conjuntiva (19/232). Nos felinos os três principais tumores foram o CCE $(10 / 33)$, o hemangiossarcoma (3/33) e o mastocitoma (3/33). Ainda, os três locais mais afetados nos gatos foram a pálpebra (20/33), a órbita (5/33) e a 3 a pálpebra (4/33). 0 Quadro 3 mostra a distribuição das duas principais neoplasias caninas (adenoma meibomiano e melanoma) conforme as raças de cães de maior ocorrência (Poodle, Labrador e Dachshund).

Na Figura 1 estão ilustrados cortes sagitais de globos oculares de cães e gatos com tumores diagnosticados pelo SPV-UFRGS. Estão demonstrados o melanoma conjuntival em um canino (Fig.1A) caracterizado por uma massa irregular enegrecida sobre a conjuntiva bulbar se estendendo até a córnea, o fibrossarcoma orbital em um felino (Fig.1B) onde observa-se uma massa brancacenta localizada lateralmente ao bulbo ocular, o meningioma orbital em região retrobulbar de um canino (Fig.1C) caracterizado por uma massa brancacenta irregular e um adenoma iridociliar em um canino (Fig.1D), onde observa-se um nódulo brancacento aderido a íris e corpo ciliar se estendendo a lateral do cristalino.

\section{DISCUSSÃO}

A maior parte dos cães e gatos acometidos por neoplasias oculares e dos anexos, neste estudo, eram adultos e idosos, a média de idade nos caninos concorda com a observada no trabalho de Olbertz \& Montiani-Ferreira (2012) que foi de 8,1 anos. Em caninos a frequência de machos e fêmeas afetados por neoplasias foi semelhante e em felinos observou-se maior frequência de fêmeas $(66,67 \%)$ em relação a machos $(33,33 \%)$, porém não é conhecido o número total de machos e fêmeas na população geral. Nos estudos realizados por Olbertz \& Montiani-Ferreira (2012) e Dubielzig (2011) não foram encontrados dados que indicassem que o sexo dos cães e gatos é um fator de risco para o aparecimento de neoplasias oculares. No trabalho de Silva (2013), os cães e gatos machos foram mais frequentemente acometidos do que as fêmeas. No caso dos gatos, estudos recentes identificaram uma prevalência superior de sarcoma pós-traumático em machos, em detrimento das fêmeas (Dubielzig et al. 2010), contudo no presente trabalho houve somente um caso de sarcoma pós-traumático.

Os cães e gatos sem raça definida foram os mais afetados, dado atribuído possivelmente ao fato de estes representarem a maioria das amostras encaminhadas ao SPV-UFRGS. 
Quadro 1. Distribuição dos neoplasmas oculares em cães de acordo com os diagnósticos histopatológicos e localização

\begin{tabular}{|c|c|c|c|c|c|c|c|c|c|}
\hline Classificação histopatológica & Pálpebra & 3a Pálpebra & Conjuntiva & Bulbo ocular & Órbita & Úvea & Córnea & Total & $\%$ \\
\hline Adenoma meibomiano & 82 & - & - & - & - & - & - & 82 & 35,35 \\
\hline Melanoma maligno & 19 & 3 & 2 & 9 & - & 1 & 1 & 35 & 15,09 \\
\hline Hemangiossarcoma & 3 & 8 & 4 & - & - & - & - & 15 & 6,47 \\
\hline Epitelioma meibomiano & 14 & - & - & - & - & - & - & 14 & 6,03 \\
\hline $\mathrm{CCE}^{\mathrm{a}}$ & 7 & 1 & 1 & - & - & - & 3 & 12 & 5,17 \\
\hline Melanocitoma & 8 & 2 & 1 & - & - & 1 & - & 12 & 5,17 \\
\hline Papiloma & 6 & - & 4 & - & - & - & - & 10 & 4,31 \\
\hline Adenocarcinoma meibomiano & 7 & - & - & - & - & - & - & 7 & 3,02 \\
\hline Histiocitoma & 2 & 2 & 2 & - & - & - & - & 6 & 2,59 \\
\hline Hemangioma & 2 & 1 & 2 & - & - & - & - & 5 & 2,16 \\
\hline Mastocitoma & 4 & - & 1 & - & - & - & - & 5 & 2,16 \\
\hline Meningioma & - & - & - & - & 5 & - & - & 5 & 2,16 \\
\hline $\begin{array}{l}\text { Adenocarcinoma de } \\
\text { glândula de } 3^{a} \text { pálpebra }\end{array}$ & - & 4 & - & - & - & - & - & 4 & 1,72 \\
\hline Fibrossarcoma & 3 & - & - & - & 1 & - & - & 4 & 1,72 \\
\hline Adenoma iridociliar & - & - & - & - & - & 3 & - & 3 & 1,29 \\
\hline Sarcoma histiocítico & 1 & - & 1 & - & 1 & - & - & 3 & 1,29 \\
\hline Carcinoma de células basais & 2 & - & - & - & - & - & - & 2 & 0,86 \\
\hline Fibroma & 2 & - & - & - & - & - & - & 2 & 0,86 \\
\hline Carcinoma iridociliar & - & - & - & - & - & 1 & - & 1 & 0,43 \\
\hline Carcinoma retrobulbar & - & - & - & - & 1 & - & - & 1 & 0,43 \\
\hline Linfangioma & 1 & - & - & - & - & - & - & 1 & 0,43 \\
\hline Linfoma & - & - & - & 1 & - & - & - & 1 & 0,43 \\
\hline Pilomatricoma & 1 & - & - & - & - & - & - & 1 & 0,43 \\
\hline $\mathrm{TVT}^{\mathrm{b}}$ & - & - & 1 & - & - & - & - & 1 & 0,43 \\
\hline TOTAL & 164 & 21 & 19 & 10 & 8 & 6 & 4 & 232 & 100 \\
\hline$\%$ & 70,7 & 9,1 & 8,2 & 4,5 & 3,2 & 2,6 & 1,7 & 100 & \\
\hline
\end{tabular}

$\overline{{ }^{a}}$ CCE = carcinoma de células escamosas, ${ }^{\mathrm{b}}$ Tumor venéreo transmissível.

Quadro 2. Distribuição dos neoplasmas oculares em gatos de acordo com os diagnósticos histopatológicos e localização

\begin{tabular}{|c|c|c|c|c|c|c|c|c|c|}
\hline Classificação histopatológica & Pálpebra & 3a Pálpebra & Conjuntiva & Bulbo ocular & Órbita & Úvea & Córnea & Total & $\%$ \\
\hline $\mathrm{CCE}^{\mathrm{a}}$ & 7 & 3 & - & - & - & - & - & 10 & 30,31 \\
\hline Hemangiossarcoma & 2 & 1 & - & - & - & - & - & 3 & 9,09 \\
\hline Mastocitoma & 3 & - & - & - & - & - & - & 3 & 9,09 \\
\hline Fibrossarcoma & 1 & - & - & - & 1 & - & - & 2 & 6,06 \\
\hline Linfoma & - & - & - & - & 2 & - & - & 2 & 6,06 \\
\hline Melanoma & - & - & - & 1 & - & 1 & - & 2 & 6,06 \\
\hline Meningioma & - & - & - & - & 2 & - & - & 2 & 6,06 \\
\hline Adenoma apócrino ductular & 1 & - & - & - & - & - & - & 1 & 3,03 \\
\hline $\begin{array}{l}\text { Adenoma cistíco de } \\
\text { glândula sudorípara }\end{array}$ & 1 & - & - & - & - & - & - & 1 & 3,03 \\
\hline Adenoma iridociliar & - & - & - & - & - & 1 & - & 1 & 3,03 \\
\hline Adenoma meibomiano & 1 & - & - & - & - & - & - & 1 & 3,03 \\
\hline Fibroma & 1 & - & - & - & - & - & - & 1 & 3,03 \\
\hline Melanocitoma & - & - & - & - & - & 1 & - & 1 & 3,03 \\
\hline Sarcoma pós-traumático & 1 & - & - & - & - & - & - & 1 & 3,03 \\
\hline $\begin{array}{l}\text { Tumor de bainha de nervo } \\
\text { periférico benigno }\end{array}$ & 1 & - & - & - & - & - & - & 1 & 3,03 \\
\hline Tumor de células basais & 1 & - & - & - & - & - & - & 1 & 3,03 \\
\hline TOTAL & 20 & 4 & 0 & 1 & 5 & 3 & 0 & 33 & 100 \\
\hline$\%$ & 60,6 & 12,1 & 0,0 & 3,0 & 15,2 & 9,1 & 0,0 & 100 & \\
\hline
\end{tabular}

${ }^{a}$ CCE = carcinoma de células escamosas.

Gatos da raça Persa e Siamesa foram os mais acometidos, concordando com Silva (2013). No estudo realizado por Olbertz \& Montiani-Ferreira (2012), igual ao presente trabalho, a raça Poodle foi a mais afetada, o mesmo autor avaliou ainda, qual a neoplasia mais observada nas principais raças do estudo. Os cães da raça Poodle foram acometidos principalmente por epitelioma meiobomiano (Olbertz \& Montiani-Ferreira 2012), já no atual trabalho houve maior frequência de adenomas meibomianos nesta raça.
As neoplasias primárias do olho e seus anexos corresponderam à grande parte dos casos, em ambas as espécies. Os tumores cuja origem ocorre em outro sítio, que não no globo ocular, são ditos neoplasias secundárias, que neste estudo foi representado, nos cães e gatos, pelo linfoma e pelo sarcoma histiocítico, somente nos cães. Porém no histórico dos animais, não foi especificada a origem desses tumores. 0 linfoma é a neoplasias secundária intra-ocular mais comum em cães e gatos, podendo ser também de ori- 
gem primária (Miller \& Dubielzig 2007). O sarcoma histiocítico é uma neoplasia canina sistêmica, na qual a doença ocular pode ser a apresentação inicial. 0 envolvimento ocular normalmente é unilateral (Dubielzig et al. 2010).

Os felinos apresentaram maior número de casos de neoplasias malignas em comparação com os caninos, os quais tiveram um maior número de diagnósticos de neoplasias benignas, resultados estes que se assemelham ao demonstrado no estudo realizado por Silva (2013).

A pálpebra foi o local mais acometido na espécie canina e felina. Williams (1981) e Newkirk \& Rohrbach (2009) corroboram com nossos achados quanto à locali-

\section{Quadro 3. Distribuição dos principais neoplasmas oculares e de anexos dos caninos conforme as raças de maior ocorrência}

\begin{tabular}{ccc}
\hline $\begin{array}{c}\text { Classificação } \\
\text { histopatológica }\end{array}$ & Raças & $\begin{array}{c}\text { № casos/no de } \\
\text { cães na raça }\end{array}$ \\
\hline Adenoma meibomiano & Poodle & $10 / 21$ \\
& Labrador & $9 / 19$ \\
Melanoma & Dachshund & $6 / 14$ \\
& Dachshund & $4 / 21$ \\
& Labrador & $3 / 19$ \\
& Poodle & $1 / 14$
\end{tabular}

zação, porém citam este achado mais prevalente na espécie canina.

De acordo com estudos retrospectivos a maioria dos tumores de pálpebra em cães são benignos (Krehbiel 1975, Roberts 1986). Nos felinos, ao contrário dos caninos, a maioria das neoplasias em pálpebras são malignas (Williams 1981, Newkirk \& Rohrbach 2009). Esta afirmação corrobora com os dados obtidos nesse trabalho, onde as quatro primeiras neoplasias são de natureza maligna e estão localizadas, principalmente, em pálpebra.

Nos demais locais, os gatos tiveram como região mais afetada a órbita, a $3^{3}$ pálpebra e a úvea e nos cães o sítio de maior ocorrência foram a $3^{\mathrm{a}}$ pálpebra, a conjuntiva e o bulbo ocular. Dados estes que discordam parcialmente do estudo realizado por Silva (2013) no qual os locais mais afetados em cães foram a pálpebra e a órbita e em gatos 0 bulbo ocular, a pálpebra e a íris.

Dentre os tumores palpebrais nos cães, o adenoma meibomiano (tarsal) foi o mais observado, corroborando com os dados da literatura (Wilcock et al. 2002, Souza et al. 2005, Grahn \& Peiffer 2007, Montiani-Ferreira et al. 2008). Indo contra os dados apresentados, o trabalho realizado por Olbertz \& Montiani-Ferreira (2012), os quais avaliaram
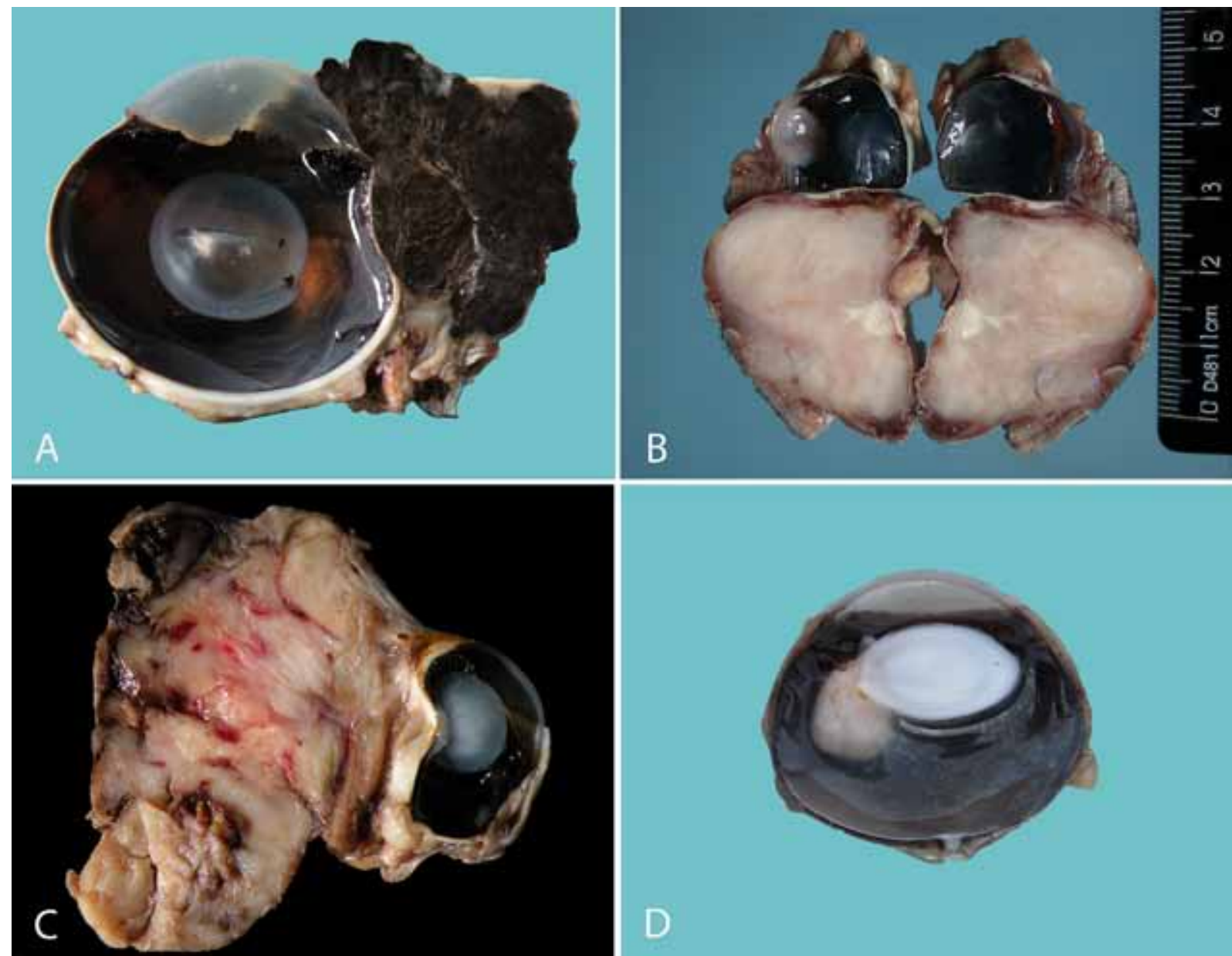

Fig.1. (A) Corte sagital de globo ocular de canino, Cocker Spaniel Inglês, 11 anos com melanoma conjuntival. (B) Corte sagital de globo ocular de felino, SRD, 13 anos com fibrossarcoma orbital. (C) Corte sagital de globo ocular de canino, Buldogue Campeiro, 11 anos com meningioma orbital (D) Corte sagital de globo ocular de canino, Poodle, 8 anos com adenoma iridociliar. 
somente a ocorrência de adenomas e epiteliomas meibomianos nas pálpebras de cães, o epitelioma foi muito mais frequente. Ainda, os mesmos autores não citam razão para esta diferença.

De acordo com Morgan (1969) e Dubielzig (2002) os tumores de origem melanocíticas são os tumores primários mais comuns do globo ocular de caninos. Informação que corrobora com os dados obtidos neste estudo, mas discorda de Wilcock \& Peiffer (1986), os quais afirmam que o melanoma intraocular compreende cerca de $20 \%$ dos tumores melanocíticos intraoculares em cães. Nos felinos, os tumores primários intraoculares melanocíticos são também considerados comuns (Dubielzig 1997), dado observado neste estudo. Na literatura (Dubielzig 2002), há uma classificação conforme a localização dos tumores melanocíticos no globo ocular, e esta pode predizer um maior ou menor grau a malignidade do tumor. Os melanocitomas podem ocorrer em vários locais, os mais comuns são no trato uveal anterior, na íris ou corpo ciliar, assim como os tumores em região de limbo e esclera (epibulbar) e estes de modo geral são benignos (Dubielzig 2002). No estudo, ao considerar somente o globo ocular, observou-se nos cães o melanoma localizado principalmente no bulbo ocular, sem a identificação da origem e o melanocitoma em conjuntiva e úvea.

Ao analisar os tumores com origem no endotélio vascular, o hemangiossarcoma foi o terceiro neoplasma mais observado nos cães $(6,47 \%)$ e em menor número os hemangiomas $(2,16 \%)$, enquanto que os resultados obtidos por Dubielzig et al. (2010), mostraram que 65\% dos tumores com origem no endotélio vascular correspondiam ao hemangioma e 35\% ao hemangiossarcoma. A exposição à luz ultravioleta parece ser um fator de risco (Martin 2010). Deste modo, as raças de grande porte utilizadas para caça e atividades desportivas estão normalmente sobre-representadas, visto que estes cães são normalmente mantidos ao ar livre (Dubielzig et al. 2010). As principais raças caninas afetadas pelo hemangiossarcoma neste estudo foram $o$ Pointer, o Dogue Alemão e o São Bernardo.

O carcinoma de células escamosas (CCE) foi a neoplasia mais observada nos felinos, com localização predominante em pálpebra e terceira pálpebra, dado este que concorda com Degorge \& Parodi (1990) e McLaughlin et al. (1993). Miller \& Dubielzig (2007) relatam que este tumor constitui mais de dois terços dos tumores palpebrais e de terceira pálpebra nos gatos. Ainda, Miller \& Dubielzig (2007), caracterizam o CCE como tumores superficiais, mas que ao invadir a placa actínica podem metastizar para tecidos mais profundos. Embora raramente ocorra, há relatos de CCE com metástase para órgãos distantes (Miller \& Dubielzig 2007). Tanto em cães quanto em gatos o aumento da exposição à radiação solar, a ausência de pigmentação e doenças irritativas crônicas destes anexos são considerados fatores predisponentes para esse tumor (Krehbiel \& Langham 1975, Barrie et al. 1982, Bernays et al. 1999). Porém, neste trabalho não houve tantos casos de CCE nos caninos, o que contraria os resultados encontrados por Andrade et al. (2012) em que na Paraíba o CCE cutâneo foi mais observado, tanto em cães como em gatos. Segundo Meirelles et al. (2010), no Rio Grande do Sul a neoplasia mais prevalente na pele de cães da região metropolitana de Porto Alegre foi o mastocitoma, em seguida o CCE. Comparando esses dados dos extremos do Brasil, sugere-se que a condição climática relacionada com a incidência de radiação solar no decorrer do ano influencia no tipo de neoplasia presente na pele dos animais.

O hemangiossarcoma e o mastocitoma foram os tumores mais frequentes nos gatos, depois do CCE. No estudo realizado por Newkirk \& Rohrbach (2009) o mastocitoma foi o segundo mais frequente seguido do hemangiossarcoma. Já no trabalho de Martins \& Barros (2014), o hemangiossarcoma se apresentou em segundo lugar no número de ocorrências e não houve nenhum caso relatado de mastocitoma.

\section{CONCLUSÕES}

As biópsias oculares e de anexos recebidas pelo SPV-UFRGS durante o período de análise corresponderam a neoplasmas, localizadas principalmente em pálpebra tanto em cães como em gatos.

Os animais adultos e idosos foram os mais acometidos.

Não houve predisposição sexual em caninos, porém em felinos foi observada uma maior frequência de neoplasias em fêmeas do que em machos.

Os cães e gatos sem raça definida foram os mais acometidos, seguidos pelas raças Poodle, Labrador e Dachshund e em felinos pelas raças Persa e Siamês.

As neoplasias mais encontradas foram o adenoma meibomiano e o melanoma maligno nos caninos, e carcinoma de células escamosas e mastocitoma nos felinos.

\section{REFERÊNCIAS}

Andrade L.F.S., Oliveira D.M., Dantas A.F.M., Souza A.P., Neto P.I.N. \& Riet-Correa F. 2012. Tumores de cães e gatos diagnosticados no semiárido da Paraíba. Pesq. Vet. Bras. 32:1037-1040.

Barrie K.P., Gelatt K.N. \& Parshall C.P. 1982. Eyelid squamous cell carcinoma in four dogs. J. Am. Anim. Hosp. Assoc. 18:123-127.

Bernays M.E., Flemming D. \& Peiffer Jr R.L. 1999. Primary corneal papilloma and squamous cell carcinoma associated with pigmentary keratitis in four dogs. J. Am. Vet. Med. Assoc. 214(2):215-217.

Conceição L.F., Ribeiro A.P., Piso D.Y.T. \& Laus J.L. 2010. Considerations about ocular neoplasia of dogs and cats. Ciência Rural 40(10):22352242.

Degorge F. \& Parodi A.L. 1990. Tumeurs conjonctiveles des carnivores domestiques. Rec. Med. Vet. Special. Cancerolog. 166:1043-1060.

Dubielzig R.R. 2002. Tumors of the eye, p.739-754. In: Meuten D.J. (Ed.), Tumors in Domestic Animals. $4^{\text {th }}$ ed. Blackwell Publishing, Iowa.

Dubielzig R.R., Chappel R.J., Kalishman J.B. \& Flood L.A. 1997. Survival in cats with diffuse melanoma: a matched observational study. Proceedings of the 28th Annual Meeting of the American College of Veterinary Ophthalmology, Santa Fe, NM, p.5. (Resumo)

Dubielzig R.R., Ketring K., McLellan G.J. \& Albert D.M. 2010. Veterinary Ocular Pathology: a comparative review. Saunders Elsevier, London, p.115-418.

Dubielzig R.R. 2011. Ocular and periocular tumors in cats. Proc. $36^{\text {th }}$ World Small Animal Veterinary Congress WSAVA, Jeju, Korea, p.14-17. (Resumo)

Grahn B.H. \& Peiffer R.L. 2007. Fundamentals of veterinary ophthalmic pathology, p.355-437. In: Gelatt K.N. (Ed.), Veterinary ophthalmology. $4^{\text {th }}$ 
ed. Blackwell, Oxford.

Krehbiel J.D. \& Langham R.F. 1975. Eyelid neoplasms in dogs. Am. J. Vet. Res. 36(1):115-119.

Martin C.L. 2010. Ophthalmic Disease in Veterinary Medicine. $2^{\text {nd }}$ ed. Manson Publishing, London, p.212-213.

Martins T.B. \& Barros C.S.L. 2014. Fifty years in the blink of an eye: a retrospective study of ocular and periocular lesions in domestic animals. Pesq. Vet. Bras. 34(12):1215-1222.

McLaughlin S.A., Whitley R.D., Gilger B.C., Wright J.C. \& Lindley D.M. 1993. Eyelid neoplasms in cats: a review of demographic data (1979-1989). J. Am. Anim. Hosp. Assoc. 29:63-67.

Meirelles A.E.W.B., Oliveira E.C., Rodrigues B.A., Costa G.R., Sonne L., Tesser E.S. \& Driemeier D. 2010. Prevalência de neoplasmas cutâneos em cães da Região Metropolitana de Porto Alegre, RS: 1.017 casos (2002-2007). Pesq. Vet. Bras. 30:968-973.

Miller P.E. \& Dubielzig R.R. 2007. Ocular tumors, p.686-698. In: Withrow S.J. \& Vail D.M. (Eds). Withrow and MacEwen's Small Animal Clinical Oncology. $4^{\text {th }}$ ed. Saunders Elsevier, Saint Louis.

Montiani-Ferreira F., Kiupel M., Muzolon, P. \& Truppel J. 2008. Corneal squamous cell carcinoma in a dog: a case report. Vet. Ophthalmol. 11:269-272.

Morgan G. 1969. Ocular tumors in animals. J. Small. Anim. Pract. 10:563570.

Newkirk K.M. \& Rohrbach B.W. 2009. A retrospective study of eyelid tumors from 43 cats. Vet. Pathol. 46(5):916-927.
Olbertz L. \& Montiani-Ferreira F. 2012. Levantamento clínico-epidemiológico com análise morfológica das principais neoplasias oculares em cães. Dissertação de Mestrado do programa de Pós-Graduação do Setor de Ciências Agrárias da Universidade Federal do Paraná, Curitiba. 44p.

Roberts S.M., Severin G.A. \& Lavach J.D. 1986. Prevalence and treatment of palpebral neoplasms in the dog: 200 cases (1975-1983). J. Am. Vet. Med. Assoc. 189(10):1355-1359.

Silva B.R.F.D. 2013. Neoplasias oculares em cães e gatos: estudo retrospectivo 2001-2012. Dissertação de Mestrado Integrado, Faculdade de Medicina Veterinária, Universidade Técnica de Lisboa, Lisboa. 77p.

Souza A.L.G., Wouk A.F.P.F. \& Montiani-Ferreira F. 2005. Neoplasias dos anexos oculares em cães e gatos. Clin. Vet. 54:48-54.

Wilcock B. 2007a. Eye and ear, p.460-546. In: Maxie M.G. (Ed.), Jubb, Kennedy and Palmer's Pathology of Domestic Animals. Vol.1. $5^{\text {th }}$ ed. Elsevier, London.

Wilcock B. 2007b. Ocular and otic tumors, p.340-370. In: Baba A.I. \& Câtoi C. (Eds), Comparative Oncology. The Publishing House of the Romanian Academy, Bucharest.

Wilcock B., Dubielzig R.R \& Render J.A. 2002. Histological classification of ocular and otic tumors in domestic animals. Armed Forces Institute of Pathology, Washington, DC.

Wilcock B.P. \& Peiffer Jr R.I. 1986. Morphology and behavior of primary ocular melanomas in 91 dogs. Vet. Pathol. 23(4):418-424.

Williams L.W., Gelatt K.N. \& Gwin R.M. 1981. Ophthalmic neoplasms in the cat. J. Am. Anim. Hosp. Assoc. 17(6):999-1008. 